\title{
Effects of electron-electron interactions on the electronic Raman scattering of graphite in high magnetic fields
}

\author{
Y. Ma, ${ }^{1,{ }^{*}}$ Y. Kim,,${ }^{2,}{ }^{*}$ N. G. Kalugin, ${ }^{3}$ A. Lombardo, ${ }^{4}$ A. C. Ferrari, ${ }^{4}$ J. Kono,,${ }^{1,5}$ A. Imambekov, ${ }^{1, \dagger}$ and D. Smirnov ${ }^{2}$ \\ ${ }^{1}$ Department of Physics and Astronomy, Rice University, Houston, Texas 77005, USA \\ ${ }^{2}$ National High Magnetic Field Laboratory, Tallahassee, Florida 32310, USA \\ ${ }^{3}$ Department of Materials and Metallurgical Engineering, New Mexico Tech, Socorro, New Mexico 87801, USA \\ ${ }^{4}$ Cambridge Graphene Centre, Cambridge University, Cambridge CB3 OFA, United Kingdom \\ ${ }^{5}$ Department of Electrical and Computer Engineering, Rice University, Houston, Texas 77005, USA
}

(Received 19 April 2013; published 5 March 2014)

\begin{abstract}
We report the observation of strongly temperature $(T)$-dependent spectral lines in electronic Raman-scattering spectra of graphite in a high magnetic field up to $45 \mathrm{~T}$ applied along the $c$ axis. The magnetic field quantizes the in-plane motion, while the out-of-plane motion remains free, effectively reducing the system dimension from 3 to 1. Optically created electron-hole pairs interact with, or shake up, the one-dimensional Fermi sea in the lowest Landau subbands. Based on the Tomonaga-Luttinger liquid theory, we show that interaction effects modify the spectral line shape from $(\omega-\Delta)^{-1 / 2}$ to $(\omega-\Delta)^{2 \alpha-1 / 2}$ at $T=0$. At finite $T$, we predict a thermal broadening factor that increases linearly with $T$. Our model reproduces the observed $T$-dependent line shape, determining the electron-electron interaction parameter $\alpha$ to be $\sim 0.05$ at $40 \mathrm{~T}$.
\end{abstract}

DOI: 10.1103/PhysRevB.89.121402

PACS number(s): 78.30.Am, 71.70.Di, 76.40.+b, 78.20.Ls

Electron-electron $(e-e)$ interactions become progressively more important as the system dimension is lowered. One-dimensional (1d) systems, in particular, provide model environments in which to explore interaction effects [1]. Interacting $1 \mathrm{~d}$ electrons are expected to form an exotic electronic state of matter, the Tomonaga-Luttinger liquid (TLL) [2-5]. A strong magnetic field, $B$, can suppress the kinetic energy of electrons, thus enhancing the effect of interactions, as exemplified by the fractional quantum Hall effect [6-8]. For a 3d material, an applied magnetic field creates an effective 1d system along the field, ideal for a systematic study of interaction effects in a highly controllable fashion [9]. Particularly promising are $3 \mathrm{~d}$ metals with small electron and/or hole pockets near the Fermi energy, $E_{\mathrm{F}}$, such as bismuth [10-14] and graphite [12,15-18], where the magnetic quantum limit can be readily reached with $B \sim 10 \mathrm{~T}$.

Here we use Raman spectroscopy to study electronic states and correlations in graphite in a strong $B$ up to $45 \mathrm{~T}$ applied along the $c$ axis. The $B$ quantizes the electronic motion in the $a b$ plane while the motion along the $c$ axis remains free, thus reducing the effective dimension from 3 to 1. Instead of the main Raman features related to phonons [19,20], in this work we focus on a series of electronic Raman features assigned to electronic inter-Landaulevel (LL) transitions [21], whose $B$ dependence can be explained through the Slonczewski-Weiss-McClure (SWM) model [22-24]. Each feature exhibits strongly temperature ( $T$ )-dependent shape. Our calculations show that scattering by thermally excited acoustic phonons [25-28] is too weak to explain the observations. Electron-electron interactions, on the other hand, are shown to be the cause for the observed $T$ dependence, through the "shake-up" process, known in the problem of x-ray (or Fermi-edge) singularities [5]. Namely,

\footnotetext{
*Y. Ma and Y. Kim equally contributed to this work.

${ }^{\dagger}$ Deceased.
}

optically created electron-hole $(e-h)$ pairs interact with, or shake up, the 1d Fermi sea in the lowest Landau subbands, resulting in line-shape deviations from single-particle densities of states (i.e., 1d Van Hove singularities). Based on the TLL theory [1-5], we show that $e-e$ interactions modify the Van Hove singularity to the form $(\omega-\Delta)^{2 \alpha-1 / 2}$ at $0 \mathrm{~K}$, where $\omega$ is the photon frequency, $\Delta$ is the band-edge frequency, and $\alpha$ is a dimensionless measure of the influence of $e$ - $e$ interactions. At finite $T$, we predict a thermal broadening factor, $\Gamma(T) \propto T$. Our model reproduces the observed $T$-dependent line shape, determining $\alpha$ to be $0.016,0.026$, and 0.048 , at 20,30, and $40 \mathrm{~T}$, respectively.

Raman-scattering measurements were performed on natural graphite (NGS Naturgraphit $\mathrm{GmbH}$ ) in a back-scattering Faraday geometry in $B$ up to $45 \mathrm{~T}$, as described in Ref. [21]. The excitation beam from a 532-nm laser was focused to a spot size $<20 \mu \mathrm{m}$ with a power of $\sim 13 \mathrm{~mW}$. Most of the data were collected with a spectral resolution of $\sim 3.4 \mathrm{~cm}^{-1}$. For high- $B$, low- $T(\leqslant 10 \mathrm{~K})$ measurements of the sharpest peaks, a spectral resolution of $\sim 1.9 \mathrm{~cm}^{-1}$ was employed. The $T$ drift over an integration time of up to $7 \mathrm{~min}$, measured by a $T$ sensor installed below the sample, was $<1 \mathrm{~K}$ at $T=7 \mathrm{~K}$ and $<2 \mathrm{~K}$ at $T \geqslant 180 \mathrm{~K}$.

Figure 1(a) shows Raman spectra taken at 10, 20, and $30 \mathrm{~T}$ at $7 \mathrm{~K}$. The main band is the $G$ peak at $\sim 1580 \mathrm{~cm}^{-1}$, due to $E_{2 g}$ phonons $[19,20]$. In the presence of $B$, electronic Raman features appear, coming from inter-LL transitions, labeled $(1,1)$, $(2,2), \ldots$, etc., which we focus on in this work. Figure $1(\mathrm{~b})$ shows a series of spectra taken at various $B$ at $7 \mathrm{~K}$, exhibiting electronic peaks that move with $B$. These peaks can be attributed to the "symmetric" inter-LL excitations in the vicinity of the $K$ point [21,29]. The strongest, lowest-frequency transition among these is $(1,1)$, which is from the $n=-1$ level in the valence band to the $n=1$ level in the conduction band. Similarly, we observe the $(2,2),(3,3)$, and $(4,4)$ transitions; see also the zero-field in-plane dispersions and energy levels near the $K$ point in the inset to Fig. 1(a). The symmetric inter-LL 

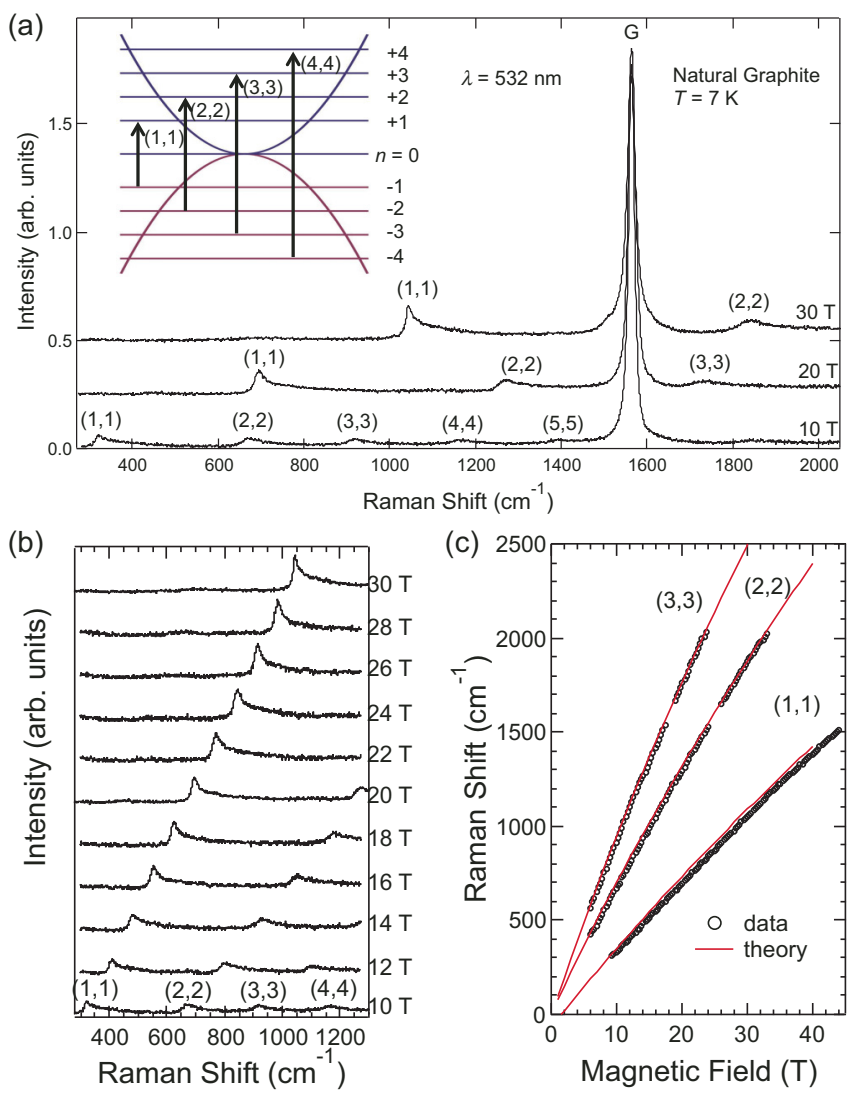

FIG. 1. (Color online) (a) Raman spectra at 10, 20, and $30 \mathrm{~T}$. The feature at $\sim 1580 \mathrm{~cm}^{-1}$ is the $G$ peak due to the $E_{2 g}$ phonons. Inset: schematic energy-level diagram showing the transitions responsible for the electronic peaks. (b) Data taken at various $B$ at $T=7 \mathrm{~K}$, showing peaks due to $(1,1)$ through $(4,4)$ interband transitions. (c) Peak positions of the observed $(1,1),(2,2)$, and $(3,3)$ transitions as a function of $B$, together with calculations based on the SWM model.

excitations are nonresonant Raman processes and were theoretically investigated for single-layer graphene (SLG) [30] and bilayer graphene (BLG) [31]. The peak positions of the three lowest-energy transitions are plotted against $B$ in Fig. 1(c); they agree well with our calculations [32] (solid and dashed lines) based on the SWM model [21].

These inter-LL transitions have strong $T$ dependence, as shown in Fig. 2, where Raman spectra at various $T$ are plotted for (a) 20, (b) 30, and (c) $40 \mathrm{~T}$. At the lowest $T$, the peaks exhibit sharp and asymmetric line shapes, reminiscent of a 1d Van Hove singularity, as expected from the effective dimension reduction from 3 to 1 in a $B$. As $T$ increases, there is significant peak broadening and blueshift. The blueshift is expected from the thermal expansion of the carbon-carbon bonds, which changes the tight-binding parameters [28]. On the other hand, the thermal broadening cannot be explained within the tight-binding model. To quantify it, we first fit the spectra within a single-particle model using the joint density of states for interband transitions, obtained from the SWM model, with $T$-dependent Lorentzian broadening [32]. Figure 2(d) plots the extracted Lorentzian FWHM $\Gamma$ as a function of $T$ for 20 and $30 \mathrm{~T}$. Apart from a small finite linewidth at $T=0$,
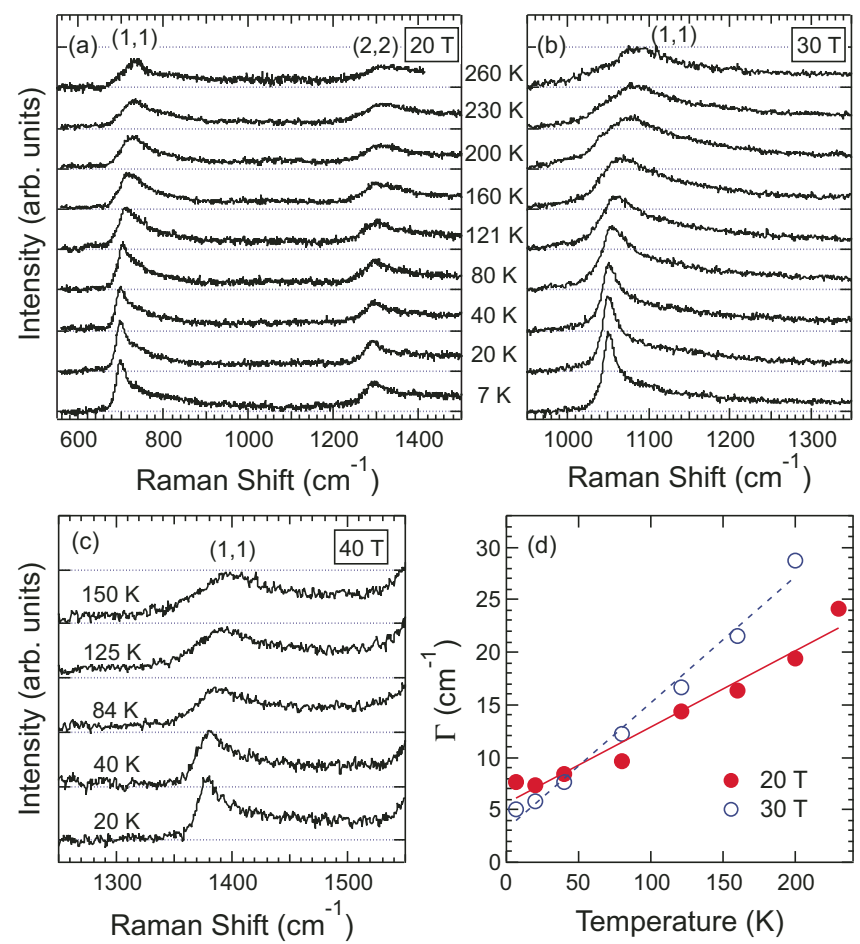

FIG. 2. (Color online) Temperature-dependent electronic Raman scattering of graphite at (a) $20 \mathrm{~T}$, (b) $30 \mathrm{~T}$, and (c) $40 \mathrm{~T}$. (d) Temperature dependence of the broadening factor $\Gamma$ of the $(1,1)$ line at $20 \mathrm{~T}$ (solid circles) and $30 \mathrm{~T}$ (open circles). The lines are fits to the data.

$\Gamma_{0} \approx 5 \mathrm{~cm}^{-1}$, possibly due to disorder, $\Gamma$ linearly depends on $T$.

Within the single-particle picture, $T$ only appears in the Fermi-Dirac distribution function, but this is a negligible effect since both the initial and final states of the Raman process are far away from $E_{\mathrm{F}}$, which resides in the $n=0$ bands. For example, for the $(1,1)$ transition at $30 \mathrm{~T}$, the electron and hole bands are $\sim 65 \mathrm{meV}$ ( or $\sim 750 \mathrm{~K}$ ) away from $E_{\mathrm{F}}$. Thus, we need to take into account the interactions of the photoexcited $e-h$ pairs with some low-energy modes that would significantly change when $T$ changes from 4 to $300 \mathrm{~K}$. Specifically, since the linear- $T$ broadening in Fig. 2(d) implies a Bose-Einstein distribution at an energy scale much smaller than $k_{\mathrm{B}} T$, we only consider bosonic excitations whose characteristic energies are $\ll 100 \mathrm{~K}$. Hence, we consider two types of low-energy modes: (i) particle-hole $(p-h)$ excitations across $E_{\mathrm{F}}$ in the $n=0$ bands [Fig. 3(a)] and (ii) acoustic phonons. We find that interactions with (i) explain the observed $T$-linear broadening while interaction with (ii) is too weak to explain it.

The magnetoelectronic Raman scattering matrix was previously calculated for SLG [30] and BLG [31] and can be readily generalized to graphite in the presence of $B$ :

$$
\hat{R}=\Lambda \sum_{\vec{k}} \Psi_{n}^{\dagger}\left(k_{y}, k_{z}\right) \Psi_{-n}\left(k_{y}, k_{z}\right),
$$

where $\Lambda$ is the scattering amplitude, $k_{y}\left(k_{z}\right)$ are electron momenta in the $a b$ plane (along the $c$ axis), $\Psi_{n}^{\dagger}$ creates an electron in the $n=1,2,3,4, \ldots$ bands, and $\Psi_{-n}$ creates a 

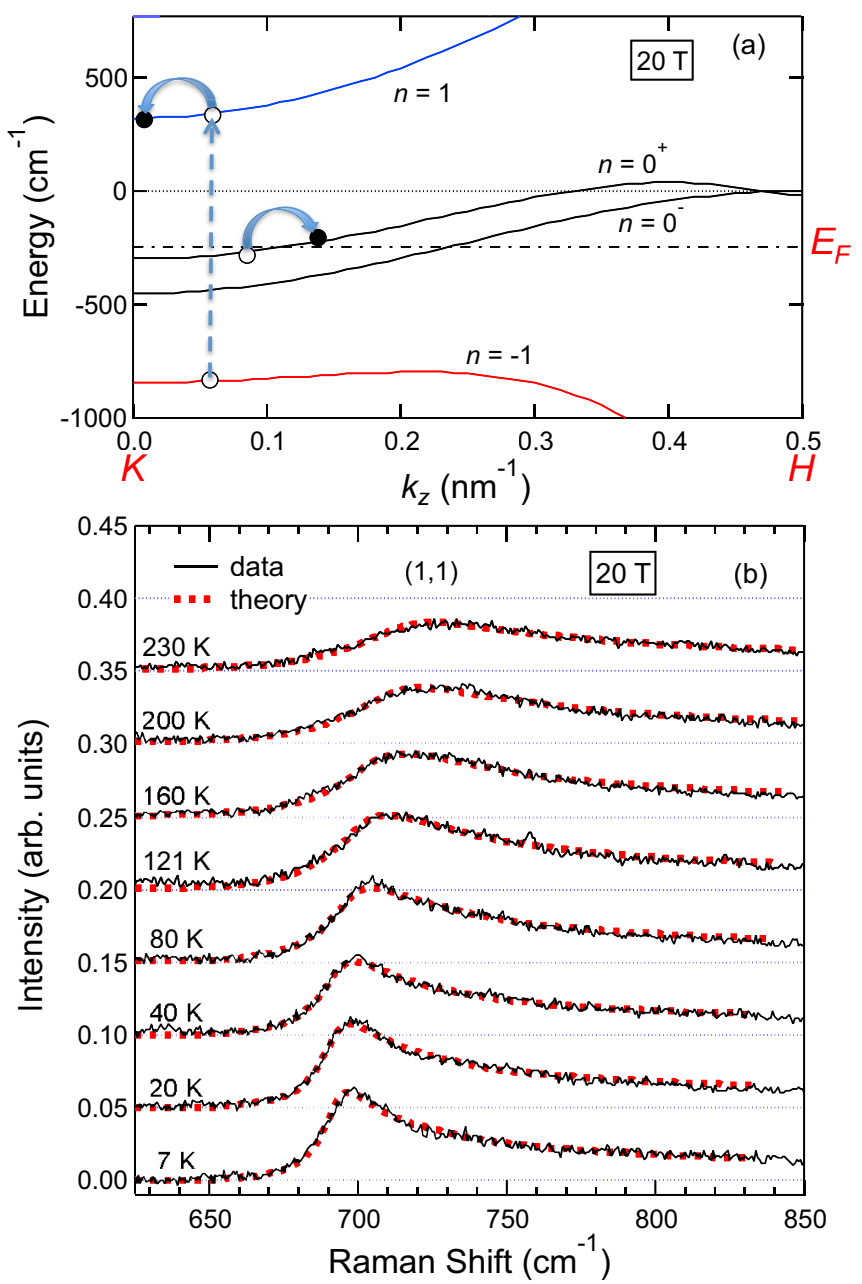

FIG. 3. (Color online) (a) The shake-up process. The $(1,1)$ electron-hole pairs shake up the 1d Fermi sea in the lowestenergy Landau subbands, creating particle-hole pairs across $E_{\mathrm{F}}$. (b) Temperature dependence of the line shape for the $(1,1)$ transition at $20 \mathrm{~T}$, together with fits (dashed lines) based on the model shown in (a).

hole in the $n=-1,-2,-3,-4, \ldots$ bands. Both electrons and holes are massive at the bottom of the bands at the $K$ point, i.e., $m_{n} \neq 0$ for all $n$ 's, similar to BLG, but there is $e$ - $h$ asymmetry, i.e., $m_{1} \neq m_{-1}$.

Figure 3(a) depicts the basic ingredients involved in the $e-e$ interaction process we consider here, together with dispersions calculated via the SWM model for the $n=0^{ \pm}$and \pm 1 bands at $20 \mathrm{~T}$. The two lowest-energy bands $\left(n=0^{ \pm}\right)$cross $E_{\mathrm{F}}$, and the carriers near $E_{\mathrm{F}}$ have approximately linear dispersions. In the $(1,1)$ process, an $e$ - $h$ pair is created in the $n= \pm 1$ bands, which interact with, and are thereby dressed with, multiple $p$ - $h$ excitations in the $n=0^{ \pm}$bands near $E_{\mathrm{F}}$. As $T$ is raised, the thermal smearing of the Fermi edge leads to stronger interaction between the massive $e-h$ pair and the massless $p-h$ pairs, and the peak broadens. This type of shake-up process was theoretically studied in carbon nanotubes at $0 \mathrm{~K}[33,34]$ : a 1d Van Hove singularity, $(\omega-\Delta)^{-1 / 2}$, is predicted to become $(\omega-\Delta)^{2 \alpha-1 / 2}$ with $\alpha \sim 0.1$ once the shake-up process is taken into account.
We describe the $n=0^{-}$electrons as a TLL with the Hamiltonian [1-4] given by

$$
H_{0}^{\mathrm{c}}=v_{\mathrm{F}} \int d z\left[\psi_{\mathrm{R}}^{\dagger} i \partial_{z} \psi_{\mathrm{R}}-\psi_{\mathrm{L}}^{\dagger} i \partial_{z} \psi_{\mathrm{L}}\right],
$$

where $v_{\mathrm{F}}$ is the Fermi velocity and $\psi_{\mathrm{R}(\mathrm{L})}^{\dagger}$ creates a particle near the right (left) Fermi point. The $n=0^{+}$band can be described by a similar Hamiltonian but with a different $v_{\mathrm{F}}$. By approximating the energy dispersion near $E_{\mathrm{F}}$ as $E \propto k_{z}$, we can rewrite Eq. (2) via bosonization as

$$
H_{0}^{\mathrm{c}}=\frac{v_{\mathrm{F}}}{2 \pi} \int d z\left[(\nabla \phi)^{2}+(\nabla \theta)^{2}\right],
$$

where $\nabla \phi=-2 \pi\left[\rho_{\mathrm{R}}+\rho_{\mathrm{L}}\right], \nabla \theta=2 \pi\left[\rho_{\mathrm{R}}-\rho_{\mathrm{L}}\right]$, and $\rho_{\mathrm{R}}$ $\left(\rho_{\mathrm{L}}\right)$ is the density operator for right-moving (left-moving) electrons.

We assume that the photogenerated electrons $(n=1)$ and holes $(n=-1)$ interact with the $n=0^{-}$conduction electrons separately. For the $n=1$ band, where electrons are massive, we can treat the electrons through

$$
H_{1}=\int d z \Psi_{1}^{\dagger}\left[-\frac{1}{2 m} \partial_{z}^{2}+\Delta_{1}\right] \Psi_{1},
$$

where $\Delta_{1}$ is the band-edge frequency and $\Psi_{1}^{\dagger}\left(\Psi_{1}\right)$ is the creation (annihilation) operator for the $n=1$ band. We also assume that the interaction Hamiltonian only involves the total charge density, thus neglecting any backscattering and umklapp scattering:

$$
H_{\text {int }}=\frac{V}{2} \int d z\left[\Psi_{1}^{\dagger} \Psi_{1}-\frac{1}{2 \pi} \nabla \phi\right]^{2} .
$$

We write the effective Hamiltonian for the system as the sum of Eqs. (3)-(5): $H=H_{0}^{\mathrm{c}}+H_{1}+H_{\text {int }}$.

The diagonalization of the Hamiltonian is a unitary transformation $U^{\dagger} H U$ and has been previously solved by many authors [33-36]:

$$
U^{\dagger}=\exp \left[-i \frac{\gamma^{+}}{\pi} \int d y \theta(y) \Psi_{1}^{\dagger}(y) \Psi_{1}(y)\right] .
$$

Under this transformation, the original interacting system can be mapped to a noninteracting one, and the massiveelectron operator acquires an additional string operator, $\Psi_{1}(z)=\exp \left[-i \gamma^{+} \theta(z) / \pi\right] \tilde{\Psi}_{1}(z)$, where $\tilde{\Psi}_{1}^{\dagger}$ creates a free electron in the $n=1$ band. The massive $n=1$ electron then gets dressed by the additional string operator, i.e., the $n=0^{-}$conduction electrons adiabatically adjust to the massive electrons. Similarly, we can obtain a dressed expression for the massive hole.

The spectral function can be obtained by calculating the imaginary part of the retarded Green's function [5],

$G^{\mathrm{R}}(z, t) \equiv-i \theta(t)\left\langle\left[\Psi_{-1}^{\dagger}(z, t) \Psi_{1}(z, t), \Psi_{1}^{\dagger}(0,0) \Psi_{-1}(0,0)\right]\right\rangle$.

At zero $T$, Eq. (7) can be evaluated directly in real space. However, at finite $T$, one has to follow a different route. As the Green's function for the massive electron/hole and that for the conduction electrons are both straightforward to obtain, 
the total Green's function can be written as a convolution of three Green's functions,

$$
\begin{aligned}
G^{\mathrm{R}}(z, t) \approx & -i \theta(t)\left[-i G_{-1}^{<}(-z,-t)\right]\left[i G_{1}^{>}(z, t)\right] F(z, t), \\
G^{\mathrm{R}}(0, \omega)= & -i \int \prod \frac{d p_{i}}{2 \pi} \frac{d \omega_{i}}{2 \pi} G^{0}\left(p_{2}, \omega_{2}\right) F\left(p_{1}, \omega_{1}\right) \\
& \times \delta\left(0-p_{1}-p_{2}\right) \delta\left(\omega-\omega_{1}-\omega_{2}\right), \\
G^{0}(p, \omega)= & \int \frac{d p_{1}}{2 \pi} \int \frac{d \omega_{1}}{2 \pi} G_{1}^{>}\left(p_{1}, \omega_{1}\right) \\
& \times G_{-1}^{<}\left(p_{1}-p, \omega_{1}-\omega\right),
\end{aligned}
$$

where

$$
F(z, t)=\langle\exp [-i \gamma \theta(x, t)] \exp [i \gamma \theta(0,0)]\rangle .
$$

We can express the spectral function in a universal form as

$$
A(\omega)=\Lambda T^{2 \alpha-0.5} \tilde{F}\left(\frac{\omega / T}{4 \pi}, \alpha\right),
$$

where

$$
\begin{aligned}
\tilde{F}(z, t)= & \sum_{n=0}^{\infty} \sum_{m=0}^{\infty} B[n+\alpha, 1-\alpha] B[m+\alpha, 1-\alpha] \\
& \times \operatorname{Re}\left[\frac{(2 i)^{2 \alpha}}{\sqrt{z-\frac{i}{2}(m+n+\alpha)}}\right] .
\end{aligned}
$$

In Eq. (11) there are two dimensionless parameters: $\omega / T$ and $\alpha$. The first parameter implies that the spectral width linearly depends on $T$ for a fixed $\alpha$. The meaning of $\alpha$ can be understood by studying the $T=0$ asymptotic behavior of Eq. (11), and comparing it with the previous zero- $T$ results $[33,34]$. It then becomes clear that

$$
A(\omega) \propto \frac{\Theta(\omega-\Delta)}{(\omega-\Delta)^{2 \alpha-1 / 2}},
$$

where $\Theta$ is the Heaviside function. For metallic carbon nanotubes, $\alpha$ was estimated to be $\sim 0.1[33,34]$. To fit our experimental data with our model, we use the true band structure instead of a parabolic approximation, by fitting the tail up to $\sim 0.2(\pi / c)$ from the $K$ point. Figure $3(\mathrm{~b})$ shows how well our model fits the data, determining $\alpha(20 \mathrm{~T}) \approx 0.016, \alpha(30 \mathrm{~T})$ $\approx 0.026$, and $\alpha(40 \mathrm{~T}) \approx 0.048$. These values are smaller than the value estimated for nanotubes, as expected, but there is a trend that $\alpha$ increases with $B$, as this tends to make the system more $1 \mathrm{~d}$.

We now consider acoustic phonons, which can also couple to the massive electrons and holes. We use the approximation that in-plane and out-of-plane modes are separated. This approximation leads to a slight numerical modification of the following equations but greatly simplifies our understanding of electron-acoustic phonon interactions in graphite. The properties of acoustic phonons can be described by five elastic constants [37]: $C_{11}=1109 \mathrm{GPa}, C_{66}=485 \mathrm{GPa}, C_{33}=$ $38.7 \mathrm{GPa}, C_{13}=0 \mathrm{GPa}$, and $C_{44}=5 \mathrm{GPa}$. Unlike the case of optical phonons [19,26,38,39], coupling with acoustic phonons vanishes at the $\Gamma$ point since the electron-acousticphonon interaction Hamiltonian $H_{\text {ep }} \sim \sqrt{q}$ [25,40,41], where $q$ is the phonon wavenumber. We then evaluate the thermal broadening of Raman peaks by calculating the imaginary part of the self-energy:

$$
\begin{aligned}
H_{\mathrm{ep}} & =\sum_{\vec{k}^{\prime}, \vec{k}, \vec{q}} g_{\vec{q}} h(\vec{q}) \Psi_{1}^{\dagger}\left(k_{y}+q_{y}, k_{z}+q_{z}\right) \Psi_{1}\left(k_{y}, k_{z}\right)\left(b_{-\vec{q}}^{\dagger}+b_{\vec{q}}\right), \\
g_{\vec{q}} & =\frac{\eta \kappa q \sin \theta}{2} \sqrt{\frac{\hbar}{2 N M \omega_{\vec{q}}}} \frac{\sqrt{2}}{2} \frac{\Delta_{B}^{2}}{\Gamma \gamma_{1}}, \\
h(\vec{q}) & =\left(4-2 l_{B}^{2} q^{2} \sin ^{2} \theta+\frac{l_{B}^{4} q^{4} \sin ^{4} \theta}{8}\right) e^{-\left(l_{B}^{2} q^{2} \sin ^{2} \theta\right) / 4},
\end{aligned}
$$

where $l_{B}=(\hbar / e B)^{1 / 2}$ is the magnetic length, $\eta \sim 2$, and $\kappa \sim$ $1 / 3$ [26]. To first order, we estimate the scattering rate through Fermi's "golden rule":

$$
W_{i}=\frac{2 \pi}{\hbar} \sum_{f}\left|\left\langle f\left|H_{\mathrm{ep}}\right| i\right\rangle\right|^{2} \delta\left(E_{i}-E_{f}\right) .
$$

When the momentum transfer during the scattering process is small (i.e., $v q \ll k_{\mathrm{B}} T$ ), the phase space for phonon modes is $q^{2}\left(\frac{1}{e^{v q / k T}}+\frac{1}{2} \pm \frac{1}{2}\right) \sim q T \rightarrow 0$, and when the momentum transfer is large, the overlap between initial and final states has a factor $\exp \left(-q_{\perp}^{2} l_{B}^{2}\right)$. For $B=30 \mathrm{~T}, l_{B} \sim 5 \mathrm{~nm}$, which is at least one order larger than the carbon-carbon bond length. Thus, the contribution to scattering drops exponentially as the phonon modes move away from the $\Gamma$ point (or, equivalently, with increasing energy). The calculated momentum-dependent scattering rate is then given by

$$
\begin{aligned}
W\left(k_{z}\right)= & \Lambda^{\prime} \int_{0}^{\pi} d \theta \frac{\tilde{q}^{2} \sin ^{3} \theta}{\sqrt{\sin ^{2} \theta+\frac{V_{4}^{2}}{V_{1}^{2}} \cos ^{2} \theta}} \frac{h^{2}(q, \theta)}{\cos ^{2} \theta} \\
& \times\left(n_{\omega_{\vec{q}}}+\frac{1}{2} \pm \frac{1}{2}\right),
\end{aligned}
$$

where

$$
\begin{aligned}
\tilde{q} & =\frac{2 m l_{B} V_{1}}{\hbar} \frac{\frac{\hbar k_{z}}{m V_{1}} \cos \theta \mp \sqrt{\sin ^{2} \theta+V_{4}^{2} / V_{1}^{2} \cos ^{2} \theta}}{\cos ^{2} \theta}, \\
\hbar \Lambda^{\prime} & =\frac{\eta^{2} \kappa^{2}}{16 \pi} \frac{m}{M} \frac{V_{\text {unit }}}{l_{B}^{3}} \frac{\Delta_{B}^{2}}{\gamma_{1}^{2}} \frac{\sqrt{2} v}{V_{1}} \Delta_{B} \approx 4.1 \times 10^{-6} \mathrm{~cm}^{-1} .
\end{aligned}
$$

This value leads to $W\left(k_{z}\right) \sim 10^{-4} \mathrm{~cm}^{-1}$ at $30 \mathrm{~T}$ and $200 \mathrm{~K}$, too small to explain the observed broadening, which requires the scattering rate to be $\sim 10 \mathrm{~cm}^{-1}$. There are two reasons for the small $\hbar \Lambda^{\prime}$ : one is $m / M \sim 10^{-3}$, and the other is $V_{\text {unit }} / l_{B}^{2}$. The latter, i.e., the magnetic length suppression, is a unique aspect of this work, made possible by a high $B$.

In summary, we studied electronic Raman scattering in graphite in a strong magnetic field up to $45 \mathrm{~T}$, applied along the $c$ axis. We observe a series of spectral lines, ascribed to inter-Landau-subband transitions, and each line exhibits strongly $T$-dependent line shape. We developed a microscopic model based on the Tomonaga-Luttinger theory, with which we show that the shake-up process can explain the observed results. Specifically, electron-electron interactions modify the Van Hove singularity to the form $(\omega-\Delta)^{2 \alpha-1 / 2}$ at $T=0$. Our model accurately reproduces the observed $T$-dependent line shape, determining $\alpha$ to be $0.016,0.026$, and 0.048 , at 20, 30, and $40 \mathrm{~T}$, respectively. 
We acknowledge funding from NHMFL UCGP-5068, DOE/BES DE-FG02-07ER46451, NSF (Grant No. DMR1006663), DOE BES Program (Grant No. DE-FG0206ER46308), the Robert A. Welch Foundation (Grant No. C1509), a Royal Society Wolfson Research Merit Award, the Eu- ropean Research Council Grants NANOPOTS and Hetero2D, EU Grants CareRAMM and Graphene Flagship (Contract no. 604391), EPSRC Grants EP/K01711X/1 and EP/K017144/1, Nokia Research Centre, Cambridge. We thank M. S. Foster, E. G. Mishchenko, and O. A. Starykh for valuable discussions.
[1] T. Giamarchi, Quantum Physics in One Dimension (Oxford University Press, Oxford, 2004).

[2] S. Tomonaga, Prog. Theor. Phys. 5, 544 (1950).

[3] J. M. Luttinger, J. Math. Phys. 4, 1154 (1963).

[4] F. D. M. Haldane, Phys. Rev. Lett. 47, 1840 (1981).

[5] G. D. Mahan, Many-Particle Physics, 3rd ed. (Kluwer Academic/Plenum, New York, 2000).

[6] D. C. Tsui, H. L. Stormer, and A. C. Gossard, Phys. Rev. Lett. 48, 1559 (1982).

[7] X. Du, I. Skachko, F. Duerr, A. Luican, and E. Y. Andrei, Nature (London) 462, 192 (2009).

[8] K. I. Bolotin, F. Ghahari, M. D. Shulman, H. L. Stormer, and P. Kim, Nature (London) 462, 196 (2009).

[9] C. Biagini, D. L. Maslov, M. Y. Reizer, and L. I. Glazman, Europhys. Lett. 55, 383 (2001).

[10] S. Mase and T. Sakai, J. Phys. Soc. Jpn. 31, 730 (1971).

[11] S. Nakajima and D. Yoshioka, J. Phys. Soc. Jpn. 40, 328 (1976).

[12] X. Du, S.-W. Tsai, D. L. Maslov, and A. F. Hebard, Phys. Rev. Lett. 94, 166601 (2005).

[13] K. Behnia, L. Balicas, and Y. Kopelevich, Science 317, 1729 (2007).

[14] L. Li, J. G. Checkelsky, Y. S. Hor, C. Uher, A. F. Hebard, R. J. Cava, and N. P. Ong, Science 321, 547 (2008).

[15] Y. Iye, P. M. Tedrow, G. Timp, M. Shayegan, M. S. Dresselhaus, G. Dresselhaus, A. Furukawa, and S. Tanuma, Phys. Rev. B 25, 5478 (1982).

[16] D. Yoshioka and H. Fukuyama, J. Phys. Soc. Jpn. 50, 725 (1981).

[17] D. V. Khveshchenko, Phys. Rev. Lett. 87, 206401 (2001).

[18] Y. Kopelevich, B. Raquet, M. Goiran, W. Escoffier, R. R. da Silva, J. C. Medina Pantoja, I. A. Luk'yanchuk, A. Sinchenko, and P. Monceau, Phys. Rev. Lett. 103, 116802 (2009).

[19] A. C. Ferrari, Solid State Commun. 143, 47 (2007).

[20] A. C. Ferrari and D. M. Basko, Nat. Nano 8, 235 (2013).

[21] Y. Kim, Y. Ma, A. Imambekov, N. G. Kalugin, A. Lombardo, A. C. Ferrari, J. Kono, and D. Smirnov, Phys. Rev. B 85, 121403(R) (2012).
[22] J. W. McClure, Phys. Rev. 108, 612 (1957).

[23] J. C. Slonczewski and P. R. Weiss, Phys. Rev. 109, 272 (1958).

[24] J. W. McClure, Phys. Rev. 119, 606 (1960).

[25] L. M. Woods and G. D. Mahan, Phys. Rev. B 61, 10651 (2000).

[26] H. Suzuura and T. Ando, Phys. Rev. B 65, 235412 (2002).

[27] J. Jiang, R. Saito, A. Grüneis, G. Dresselhaus, and M. S. Dresselhaus, Chem. Phys. Lett. 392, 383 (2004).

[28] N. Mounet and N. Marzari, Phys. Rev. B 71, 205214 (2005).

[29] P. Kossacki, C. Faugeras, M. Kühne, M. Orlita, A. A. L. Nicolet, J. M. Schneider, D. M. Basko, Y. I. Latyshev, and M. Potemski, Phys. Rev. B 84, 235138 (2011).

[30] O. Kashuba and V. I. Fal'ko, Phys. Rev. B 80, 241404 (2009).

[31] M. Mucha-Kruczyński, O. Kashuba, and V. I. Fal'ko, Phys. Rev. B 82, 045405 (2010).

[32] See Supplemental Material at http://link.aps.org/supplemental/ 10.1103/PhysRevB.89.121402 for details of our data analysis and theoretical calculation methods.

[33] L. Balents, Phys. Rev. B 61, 4429 (2000).

[34] E. G. Mishchenko and O. A. Starykh, Phys. Rev. Lett. 107, 116804 (2011).

[35] A. Imambekov and L. I. Glazman, Phys. Rev. Lett. 102, 126405 (2009).

[36] A. Imambekov and L. I. Glazman, Science 323, 228 (2009).

[37] A. Bosak, M. Krisch, M. Mohr, J. Maultzsch, and C. Thomsen, Phys. Rev. B 75, 153408 (2007).

[38] T. Ando, J. Phys. Soc. Jpn. 75, 124701 (2006).

[39] A. C. Ferrari, J. C. Meyer, V. Scardaci, C. Casiraghi, M. Lazzeri, F. Mauri, S. Piscanec, D. Jiang, K. S. Novoselov, S. Roth, and A. K. Geim, Phys. Rev. Lett. 97, 187401 (2006).

[40] N. Bonini, M. Lazzeri, N. Marzari, and F. Mauri, Phys. Rev. Lett. 99, 176802 (2007).

[41] P. Giura, N. Bonini, G. Creff, J. B. Brubach, P. Roy, and M. Lazzeri, Phys. Rev. B 86, 121404 (2012). 